\title{
Transplante cardíaco no tratamento da miocardiopatia chagásica
}

\author{
Heart transplantation in the treatment of chagasic myocardiopathy
}

Transplante cardíaco en el tratamiento de la miocardiopatía chagásica

João Gabriel Leal Contini Sanches ${ }^{1 *}$, Juliana Sabadini², Lorena Leal Fagundes ${ }^{1}$, Luna Hussein Colombelli ${ }^{3}$, Maria Luiza Vasconcelos Montenegro ${ }^{4}$, Rafaela Abrantes da Silveira ${ }^{5}$, Rayla Pons Garcia ${ }^{6}$, Stella Leony Bueno ${ }^{7}$, Thaciane Silva de Oliveira ${ }^{7}$, Alexandre Magno Macário Nunes Soares ${ }^{8}$.

\section{RESUMO}

Objetivo: Analisar o procedimento do transplante cardíaco no tratamento da miocardiopatia chagásica levando em consideração o diagnóstico, indicação, procedimento, complicações e particularidades que abordam esse assunto, para melhor compreensão dessa técnica. Revisão bibliográfica: A doença de Chagas (DC) é causada pelo protozoário flagelado Trypanosoma cruzi, representando a terceira mais prevalente infecção parasitária no mundo. Os relatos de reativação da DC são achados comuns em condições imunossupressoras, como AIDS, doenças autoimunes, câncer e, obviamente, imunossupressão farmacológica para evitar a rejeição do transplante. Dentre as complicações peri-operatórias, salienta-se hábitos de vida destrutivos, como tabagismo e etilismo, os quais promovem o agravamento de problemas cardiovasculares. Já as complicações pós operatórias englobam as neoplasias, como o linfoma e o sarcoma de Kaposi, e a possibilidade de reativação do $T$. Cruzi, devido à imunossupressão induzida, a qual se inicia após a intervenção cirúrgica, a fim de evitar a rejeição aguda do enxerto. Considerações finais: Finalmente, salienta-se a importância de um conhecimento holístico a respeito das particularidades da enfermidade chagásica, como o risco de reinfecção em pacientes imunossuprimidos, no intuito de reduzir as chances de complicações pós-operatórias.

Palavras-chave: Transplante cardíaco, Doença de Chagas, Tratamento.

\begin{abstract}
Objective: Analyze the heart transplant procedure in the treatment of chagasic myocardiopathy taking into consideration the diagnosis, indication, procedure, complications and particularities that approach this subject, to better understand this technique. Bibliographic review: Chagas Disease is caused by the flagella protozoan Trypanosoma Cruzi, representing the third most prevalent parasitic infection in the world. The reports of $C D$ reactivation are common findings in immunosuppressive conditions such as AIDS, autoimmune diseases, cancer (and the chemotherapy used to treat it) and, of course, pharmacological immunosuppression to prevent transplant rejection. Among the peri-operative complications destructive life habits, such as smoking and alcoholism, which promote the aggravation of cardiovascular problems, are highlighted. Post-operative complications include neoplasms such as lymphoma Kaposi's sarcoma and the possibility of T. Cruzi reactivation due to induced immunosuppression which begins after surgery in order to avoid acute graft rejection. Final considerations: Finally, the importance of a holistic knowledge regarding the particularities of the chagasic disease, such as the risk of re-infection in immunosuppressed patients, is highlighted in order to reduce the chances of postoperative complications.
\end{abstract}

Keywords: Heart transplantation, Chagas disease, Treatment.

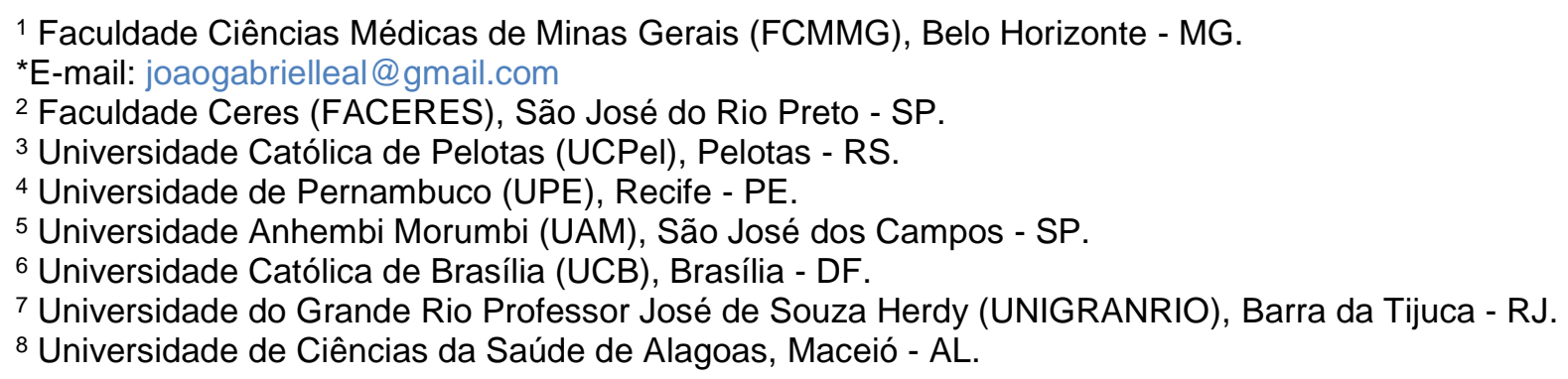




\section{RESUMEN}

Objetivo: Analizar el procedimiento de trasplante cardíaco en el tratamiento de la miocardiopatía chagásica teniendo en cuenta el diagnóstico, la indicación, el procedimiento, las complicaciones y las particularidades que abordan este tema, para comprender mejor esta técnica. Revisión bibliográfica: La enfermedad de Chagas está causada por el protozoo flagelado Trypanosoma cruzi, y representa la tercera infección parasitaria más prevalente en el mundo. Los informes de reactivación de la EC son hallazgos comunes en condiciones de inmunosupresión como el SIDA, las enfermedades autoinmunes, el cáncer (y la quimioterapia utilizada para tratarlo) y, por supuesto, la inmunosupresión farmacológica para evitar el rechazo del trasplante. Entre las complicaciones perioperatorias destacan los hábitos de vida destructivos, como el tabaquismo y el alcoholismo, que favorecen el agravamiento de los problemas cardiovasculares. Las complicaciones postoperatorias incluyen neoplasias como el linfoma y el sarcoma de Kaposi, y la posibilidad de reactivación de T. Cruzi debido a la inmunosupresión inducida, que se inicia tras la cirugía, para evitar el rechazo agudo del injerto. Consideraciones finales: Por último, se destaca la importancia de un conocimiento holístico sobre las particularidades de la enfermedad chagásica, como el riesgo de reinfección en pacientes inmunodeprimidos, para reducir las posibilidades de complicaciones postoperatorias.

Palabras clave: Transplante de corazón, Enfermedad de Chagas, Tratamiento.

\section{INTRODUÇÃO}

A Doença de Chagas (DC) é uma enfermidade severa nos países latino-americanos devido às condições determinantes para a transmissão do Trypanosoma cruzi presentes nesta região. Migrações descontroladas, degradação ambiental, alterações do clima e condições sociais e econômicas precárias contribuem para que 5.742.167 de pessoas apresentam essa condição na América Latina.Desse total, cerca de 62,4\% são residentes de Países do Cone Sul, sendo o Brasil um dos destaques dessa estatística. Ademais, é possível encontrar manifestações da infecção em regiões não endêmicas, causadas pela migração de pessoas infectadas, evidenciando, por consequência, a importância global acerca do tema (DIAS JCP, et al., 2016).

A transmissão da doença ocorre por meio do contato com o agente etiológico, seja devido a deposição do Trypanosoma cruzi sobre um ferimento causado pela picada do barbeiro, seja pelo consumo do mesmo em alimentos indevidamente armazenados e higienizados - muito comum em açaí e cana de açúcar, por exemplo (NOGUEIRA SS, et al., 2018).

O entendimento da forma de transmissão da doença de chagas é fundamental, visto que a partir dessas informações é possível planejar a profilaxia da infecção, visando reduzir o número de infectados nas regiões endêmicas. A erradicação da comorbidade em questão está entre os objetivos do Desenvolvimento do Milênio que busca "assegurar uma vida saudável e promover o bem-estar para todos" até 2030, evidenciando a importância salutar e social do combate à doença de chagas (DIAS JCP, et al., 2015).

Na doença há duas fases características: aguda e crônica. Na fase aguda, o paciente pode se apresentar assintomático ou com sinais e sintomas leves, como febre, anorexia e chagoma. Essa fase é acompanhada de altos níveis de Trypanosoma cruzi no sangue, com proliferação de amastigotas em vários tecidos (MOREIRA MC, et al., 2020; VIEIRA JF, et al., 2019).

Após a fase aguda, há a fase crônica indeterminada, a qual pode durar anos ou décadas, na qual os parasitas continuam presentes nos tecidos dos órgãos, apesar de permanecerem com poucos ou nenhum sintoma, e ainda poderem transmitir a doença. Além disso, os pacientes ao evoluírem para a fase crônica, já se apresentam com baixos níveis de parasitemia (VIEIRA JF, et al., 2019).

$\mathrm{Na}$ fase crônica sintomática, as manifestações clínicas podem aparecer mesmo décadas após a infecção inicial, ocorrendo em 20 a 30\% de todos os casos, onde a maior parte dos pacientes sofrerá danos cardíacos, como arritmias, defeitos de condução e insuficiência cardíaca, podendo evoluir também com morte súbita. Uma parte dos pacientes também desenvolvem a forma gastrointestinal, que pode resultar em megacólon ou em megaesôfago que são frequentemente associadas à forma cardíaca, constituindo assim a forma crônica mista (SIMÕES MV, et al., 2018). 
Sabe-se que a Insuficiência Cardíaca (IC) de etiologia chagásica tem um pior prognóstico e uma maior taxa de mortalidade quando comparada a outras etiologias. Nesse contexto, os casos mais graves têm como recomendação a intervenção cirúrgica utilizando do transplante cardíaco como tratamento para a respectiva complicação (BACAL F, et al., 2018).

Ademais, o resultado do transplante em pacientes com DC é melhor do que o de outras etiologias, já que geralmente os pacientes são mais jovens, possuem menos comorbidades e menores taxas de reoperações ou hipertensão pulmonar grave. Atualmente no Brasil, a miocardiopatia chagásica representa a terceira maior causa de indicação para a realização de um Transplante Cardíaco (TC) nos hospitais do país (MANGINI S, et al., 2015).

O TC é a escolha terapêutica considerada para pacientes com IC avançada e refratária à terapia medicamentosa e/ou de ressincronização cardíaca, de acordo com diretrizes nacionais e internacionais. Entretanto, sua indicação deve considerar a relação de risco-benefício individual e populacional, uma vez que os recursos são escassos e, por isso, devem ser preferencialmente ofertados ao grupo com maior probabilidade de sobrevida no longo prazo (BACAL F, et al., 2018).

Além disso, a indução da imunossupressão busca reduzir a carga gênica para diminuir a incidência de rejeição aguda e assim prolongar a sobrevida do enxerto em receptores de transplante cardíaco. Entretanto, a imunização excessiva aumenta as chances de reatividade da infecção por T. cruzi, comprometendo o tratamento. As taxas de reativação da doença em questão variam de 19,6 a $45 \%$ de acordo com as complicações presentes no quadro clínico do paciente transplantado, contudo, a mortalidade causada pela a reinfecção é de apenas $0,9 \%$ mostrando que, mesmo com a presença de uma possível complicação parasitária após o transplante a taxa de mortalidade causada por esse problema é baixa (BENATTI RD, et al., 2017; ROSCOE A, et al., 2018).

Portanto, o presente estudo tem como objetivo analisar o procedimento do transplante cardíaco no tratamento da miocardiopatia chagásica levando em consideração o diagnóstico, indicação, procedimento, complicações e particularidades que abordam esse assunto, para melhor compreensão dessa técnica, a fim de entender quais seus benefícios em longo prazo para o paciente, considerando também a possibilidade de reinfecção após a intervenção cirúrgica.

\section{REVISÃO BIBLIOGRÁFICA}

\section{Diagnósticos e indicações do transplante na doença de Chagas}

A Doença de Chagas, por ser endêmica no Brasil, é pauta de diversos estudos no país em busca de tratamentos para as complicações orgânicas da patologia, na grande maioria. Enquanto em certos indivíduos a manifestação da doença é silenciosa, em outros acomete o trato gastrointestinal e/ou, principalmente, o coração. Tendo isso em vista, o primeiro transplante cardíaco indicado para as manifestações cardiovasculares decorrentes da infecção pelo Trypanossoma cruzi foi realizado no Brasil em 1985 executado por Zerbini, proporcionando possíveis mudanças no tratamento da doença em questão. No século XXI, é a terceira indicação mais prevalente de transplante de coração no país em casos de pacientes em estado grave e avançado da patologia (DIAS JCP, et al., 2015; BACAL F, et al., 2018; BENATTI RD, et al., 2017; KRANSDORF EP, et al., 2018; KRANSDORF EP, et al., 2014).

Além da observação clínica e epidemiológica, pode-se obter o diagnóstico da doença de Chagas por meio de testes e exames. Dessa forma, o método da Reação em Cadeia da Polimerase (RCP) tem evidente sensibilidade para detecção do material genético do parasita na fase aguda da infecção, assim como a utilização de técnicas de xenodiagnóstico, biópsia de órgãos acometidos e análise de sangue - seja para cultura ou para achados parasitários. Em contrapartida, na fase crônica, os métodos com base em anticorpos e antígenos são os mais utilizados, específicos e eficientes, sendo os principais o Ensaio de Imunoabsorção Enzimática (ELISA), a imunofluorescência e a reação de hemaglutinação (BACAL $F$, et al., 2018; BENATTI RD, et al., 2017; KRANSDORF EP, et al., 2014; KRANSDORF EP, et al., 2018; SANTOS E e FALCÃO LM, 2019). 
Quanto a reativação da infecção chagásica após o transplante cardíaco, o diagnóstico se dá pela análise clínica do paciente, bem como pela existência de formas parasitárias do T. cruzi em partes do organismo, como líquido cefalorraquidiano, sangue e tecidos (BACAL F, et al., 2018; KRANSDORF EP, et al., 2014; KRANSDORF EP, et al., 2018; SANTOS E e FALCÃO LM, 2019).

Sabe-se que a cardiomiopatia chagásica corresponde a $35 \%$ das causas de transplantes cardíacos no Brasil, sendo o tratamento cirúrgico a referência para essa complicação. Outrossim, a manifestação de falência cardíaca da doença de Chagas tem como evidência fisiopatológica a miocardite, proporcionando o acometimento das câmaras cardíacas, a redução da porcentagem da fração de ejeção, tromboembolismos, arritmias cardíacas, taquicardia e infartos, podendo-se identificar em exames de monitoramento cardíaco. Assim, a indicação do transplante de coração para pacientes se dá em estágios mais evoluídos ou terminais da manifestação cardíaca da doença de Chagas. Porém, o megaesôfago decorrente da doença chagásica constitui uma contraindicação importante do transplante cardíaco, uma vez que a dilatação de parte do trato gastrointestinal anatomicamente dificulta a cirurgia e eleva as chances de intercorrências no pós-operatório (BENATTI RD, et al., 2017; KRANSDORF EP, et al., 2014; KRANSDORF EP, et al., 2018; SANTOS E e FALCÃO LM, 2019).

Em relação à taxa de sobrevivência após transplante de coração por causa da cardiomiopatia da doença de Chagas é superior ao procedimento cirúrgico realizado para outras patologias cardíacas, sendo de $71 \%$ em um ano e de $46 \%$ em dez anos após cirurgia (SANTOS E e FALCÃO LM, 2019).

\section{Procedimento Cirúrgico}

Uma vez recomendada a intervenção cirúrgica utilizando-se do transplante cardíaco, organizações nacionais, estaduais e municipais coordenam-se para garantir a distribuição de órgãos de forma efetiva para aqueles que aguardam na fila, garantindo a identificação e manutenção de potenciais doadores. Logo, uma vez obtido o consentimento familiar, inicia-se um protocolo que garante que os órgãos doados cheguem de forma segura ao seu receptor (BACAL F, et al., 2018).

No caso do transplante cardíaco, a comunidade científica tem como consenso que o tempo de isquemia fria não deve ultrapassar 4 horas, portanto, o preparo tanto do doador quanto do receptor deve ocorrer de forma simultânea. O procedimento inicia-se no doador a partir da heparinização, procedimento que irá garantir um maior tempo de coagulação sanguínea; em seguida, realiza-se a descompressão do coração através da abertura de uma das veias pulmonares, seccionando, logo após, a cava inferior ou superior. Clampeia-se a aorta ascendente, infundindo a solução cardioplégica, induzindo a parada cardíaca durante o processo, que irá prosseguir a partir de então, com a secção do restante dos vasos mais distalmente possível do coração. Feita então a retirada, o órgão é colocado em soluções que irão preservá-lo até que seja colocado em seu receptor (BACAL F, et al., 2018).

Já o procedimento no receptor pode ser feito a partir de duas técnicas: a clássica, ou mesmo, atrial. Ocorre a partir de quatro anastomoses: átrios direito e esquerdo, artéria pulmonar e aorta, ou seja, há a preservação das câmaras atriais, consequentemente, preserva-se os nós sinoatrial e atrioventricular e, também, as valvas atrioventriculares (ALMEIDA DR, 2016). Porém, observou-se com isso, uma maior incidência de insuficiência tricúspide e de arritmias atriais, fazendo com que a técnica bicaval ganhasse destaque e passasse a ser mais utilizada. Essa, por sua vez, consiste na remoção completa do coração do receptor, realizando as anastomoses dos vasos que estão conectados ao coração e ligando-os ao novo coração transplantado, essas anastomoses são realizadas a partir da avaliação do tempo de isquemia, criando-se uma sequência adequada a partir de então (BACAL F, et al., 2018).

\section{Complicações}

Sabe-se que as disfunções do aparelho circulatório são uma grande problemática do sistema de saúde no século XXI. Sendo assim, tem-se um quadro em que as doenças cardiovasculares são responsáveis em grande parte pela mortalidade global - numa proporção de 17.528 mortes em um cenário total de 35.576 pessoas. Nesse sentido, com o avanço da medicina e da tecnologia, estudos apontaram que, a partir das últimas décadas, os casos de distúrbios cardíacos graves possuem de recursos cirúrgicos para substituição de estruturas cardíacas, a exemplo o transplante de coração (BENATTI RD, et al., 2018). 
As doenças do aparelho circulatório são consideradas um grande problema atual de saúde pública. Dentre essas, as doenças cardiovasculares aparecem em primeiro lugar como responsáveis pela mortalidade de 17.528 pessoas em um total global de $\mathbf{3 5 . 5 7 6}$ mortes no mundo. Estudos mostram que, nos últimos anos, com os avanços da Medicina e da tecnologia, os casos graves, antes considerados irrecuperáveis, têm recursos de cirurgias para substituição de estruturas cardíacas, como nos transplantes cardíacos (BENATTI $\mathrm{RD}$, et al., 2018).

O conhecimento de complicações peri e pós-operatórias acontecem em pacientes indicados ao transplante cardíaco por doença de Chagas. À vista disso, ressalta-se que, no âmbito peri operatório, os maus hábitos, como tabagismo e etilismo, influenciam diretamente a qualidade de vida do receptor do órgão, e podem ser nocivos à saúde, uma vez que agravam problemas cardiovasculares, com consequente comprometimento da realização do enxerto. Outrossim, relata-se a probabilidade de ocorrer alterações no trato gastrointestinal na doença chagásica, as quais podem comprometer o resultado do transplante cardíaco, elevando os riscos de complicações peri e pós-operatórias. Por outro lado, existem obstáculos inerentes ao procedimento de transplantação, como impasses na logística de captação, baixa disponibilidade de órgãos e longa espera pelo procedimento (BACAL F, et al., 2018).

É sabido que pacientes acometidos pela doença de Chagas que sejam imunossuprimidos com o Vírus da Imunodeficiência Humana (HIV), quimioterapia, malignidades, medicações imunossupressoras para evitar a rejeição do transplante, bem como para transplantação de células hematopoiéticas, são mais suscetíveis a reinfecção chagásica pós-operatória. Esses indivíduos encontram-se com achados clínicos como febre, paniculite, pancitopenia ou miocardite. Contudo, apesar do parasita Trypanosoma cruzi ter uma alta porcentagem de reativação (cerca de 19,6 e 45\%), a taxa de mortalidade por reinfecção chagásica é apenas de 0,9\% (BENATTI RD, et al., 2017; MOREIRA MC, et al., 2020; ROSCOE A, et al., 2018).

Ademais, é válido salientar que a rejeição de transplante cardíaco como uma das complicações pósoperatórias não apresenta índice discrepante entre paciente com ou sem a doença chagásica. Relata-se que entre 15 a $60 \%$ das rejeições tardias e 5 a $36 \%$ das perdas do enxerto estão associadas à inexistência de adesão ao tratamento correto. Ainda, sabe-se que infecções ocasionais, que não se relacionem com o parasita da doença de Chagas, são a principal causa de morte para pacientes transplantados, sendo comum a infecção do trato respiratório $(40,9 \%)$ e infecção cirúrgica $(18,1 \%)$. Nesse contexto, a ocorrência de relatos infecciosos virais e bacterianos se assemelha em receptores do transplante cardíaco com ou sem a presença da doença chagásica (BENATTI RD, et al., 2017; POLTRONIERI NV, et al., 2020; RADISIC MV e REPETTO SA, 2020).

Medicamentos utilizados pós transplante a fim de diminuir a carga gênica e evitar a rejeição aguda do enxerto, bem como a doença de Chagas em si, apresentam ação imunossupressora que pode predispor o paciente ao desenvolvimento de doenças malignas, como o câncer, o qual apresenta-se como a terceira causa de morte - com porcentagem de 4,3 - em destinatários do transplante (BENATTI RD, et al., 2017). Descreve-se que existe a possibilidade de na ausência de imunossupressão, as taxas de malignidades de pacientes chagásicos ou não chagásicos se igualarem. Além disso, demonstrou-se por meio de estudos que no pós-operatório houve um aumento no número de casos de diabetes mellitus e aumento de insuficiência renal crônica (BENATTI RD, et al., 2017; POLTRONIERI NV, et al., 2020; MOREIRA MC, et al., 2020).

\section{Particularidades}

A doença de Chagas propicia aos seus pacientes, particularidades que não são encontradas em outras cardiopatias que têm a necessidade de ser abordadas com o transplante cardíaco. Dentre essas tem-se preocupação em relação à abordagem na rejeição, a complexidade de um protocolo imunossupressor adequado, à frequência de neoplasias atreladas, bem como o medo de reinfecção pelo T. cruzi (ROSCOE A, et al., 2018).

A indicação do transplante cardíaco em pacientes com insuficiência cardíaca secundária à cardiomiopatia chagásica é um tanto discutível em comparação com as cardiomiopatias não chagásicas. Isso é colocado pelo fato de a ausência de rejeição estar atrelada em $53 \%$ dos pacientes e ainda os receptores terem a 
rejeição como $10 \%$ da sua causa da morte. Contudo, ainda que se tenha esses resultados, já foi demonstrado que essa foi semelhante em transplantes cardíacos de pacientes chagásicos quando comparado com pacientes não-chagásicos (MELO CF, et al., 2020).

A imunossupressão de indução visa reduzir a carga antigênica atenuando a incidência de rejeição aguda. Essa medida visa prolongar a sobrevida do enxerto em receptores de transplante cardíaco com alto risco de rejeição. Contudo, a falta de protocolos de imunossupressão e a complexidade de individualizar para cada paciente dificulta essa abordagem. Além disso, é importante ressaltar que a imunossupressão excessiva eleva a possibilidade de reativação do T. cruzi (ROSCOE A, et al., 2018).

Acredita-se que a reativação supracitada possui relação com o desequilíbrio entre hospedeiro e parasita, no qual o hospedeiro, devido a imunossupressão causada pelos medicamentos pós-cirúrgicos, é prejudicado com a deficiência do sistema imunológico (MELO CF, et al., 2020).

Ademais, a presença do parasita em reservatórios no corpo imunossuprimido pode significar um aumento na reprodução do mesmo, contribuindo assim para a reinfecção. No primeiro ano decorrido após a realização do transplante a carga medicamentosa da classe de remédios em questão é maior, elevando, por conseguinte, as chances de ocorrência de uma possível reativação da doença de chagas. Por fim, há uma hipótese da relação entre o tipo de procedimento realizado e o ressurgimento do quadro clínico, no qual a reativação em transplantes bicavais é menos comum do que nos transplantes bitriais (RODRIGUES BA, et al., 2020).

No que diz respeito às neoplasias, foi visto que elas são a terceira causa de morte em receptores do transplante cardíaco com doença de chagas (BENATTI RD, et al., 2017). Já foi relatado que pacientes desenvolveram neoplasias como linfoma e sarcoma de kaposi no seguimento pós-transplante. Apesar desses quadros, o transplante de coração representa a terapia padrão ouro para doença de chagas em estágio final (ROSCOE A, et al., 2018; BENATTI RD, et al., 2018).

A doença de chagas, anteriormente, já foi contraindicação para o transplante cardíaco tendo em vista a possibilidade de reativação do $T$. cruzi. No entanto, a incidência de reativação da infecção por $T$. cruzi no transplante cardíaco varia de 27 a $90 \%$ e está, muitas vezes, relacionada a pacientes imunocomprometidos com neoplasia ou infecção pelo HIV (BESTETTI RB e THEODOROPOULOS T, 2009; MOREIRA MC, et al., 2020).

Ainda que a morbidade do transplante cardíaco em pacientes com doença de chagas em comparação com a do transplante para cardiomiopatias em outras etiologias (idiopática, isquêmica) seja maior, aquele tem se mostrado altamente eficaz (BESTETTI RB, 2017). Existem ainda estudos que demonstram igualdade de sobrevida pós-transplante entre pacientes com doença de chagas e pacientes com cardiomiopatia dilatada idiopática (ROSCOE A, et al., 2018). A respeito da necessidade de tratamento hospitalar para rejeição também foi semelhante para ambas etiologias (ROSCOE A, et al., 2018; BENATTI RD, et al., 2018).

Contudo, pelo fato da Doença de Chagas propiciar particularidades no que tange transplante cardíaco, é de suma importância que existam melhores registros a respeito dessa patologia para que possam ser formados mais dados concretos. Tais dados poderão elevar a eficácia do tratamento e fomentar melhores resultados aos pacientes (BENATTI RD, et al., 2018).

\section{CONSIDERAÇÕES FINAIS}

Por meio da análise bibliográfica deste estudo, pôde-se concluir que a cardiomiopatia chagásica é consequência da fase crônica de uma enfermidade prevalente nos países latino-americanos. Nos pacientes portadores de insuficiência cardíaca de etiologia chagásica, o prognóstico é mais reservado e o tratamento, por meio do transplante cardíaco, torna-se fundamental, já que a sobrevida é maior quando comparada a outros procedimentos cirúrgicos de outras patologias. Sendo assim, é imperativo conhecer os riscos e benefícios desse procedimento, pois a terapia implementada pode evitar a rejeição do órgão, mas também pode propiciar a reinfecção pelo T. Cruzi na fase pós-operatória. 


\section{REFERÊNCIAS}

1. ALMEIDA DR. Transplante cardíaco na Doença de Chagas. Revista da Sociedade de Cardiologia do Estado de São Paulo, 2016; 26(4): 266-71.

2. BACAL F, et al. 3a Diretriz Brasileira de Transplante Cardíaco. Arquivos brasileiros de cardiologia, 2018; 111(2): 230289.

3. BENATTI RD, et al. Heart Transplant for Chagas Cardiomyopathy. Journal of Heart and Lung Transplantation,2017; 36(6): 597-603.

4. BENATTI RD, et al. Heart Transplant Outcomes in patients with Chagas Cardiomyopathy in the United States. Clinical Transplantation, 2018; 32(6).

5. BESTETTI RB. Cardiomiopatia Chagásica crônica - diagnóstico e tratamento. Revista da Sociedade de Cardiologia do Estado de São Paulo, 2016; 26(4): 246-252.

6. BESTETTI RB, THEODOROPOULOS T. A Systematic Review of Studies on Heart Transplantation for Patients With End-Stage Chagas' Heart Disease. Journal of Cardiac Failure, 2009; 12(3): 249-255.

7. DIAS JCP, et al. II Consenso Brasileiro em Doença de Chagas, 2015. Epidemiologia e Serviços de Saúde, 2016; 25(21): 1-10.

8. KRANSDORF EP, et al. Chagas disease in solid organ and heart transplantation. Lippincott Williams \& Wilkins, 2014; 27(5): 1-6.

9. KRANSDORF EP, et al. Diagnosis and Management of Chagas Cardiomyopathy in the United States. Current Cardiology Reports, 2018.

10. MANGINI S, et al. Transplante cardíaco: revisão. Einstein (São Paulo), 2015; 13(2): 310-318.

11. MELO CF, et al. Entre el pulsar y el morir: la vivencia de pacientes que esperan el trasplante cardíaco. Enfermería global, 2020; (58): 351-363.

12. MOREIRA MC, CUNHA-MELO JR. Chagas disease infection reactivation after heart transplant. Tropical Medicine and Infectious Disease, 2020; 5(3): 106.

13. MOREIRA MC, et al. Heart Transplantation for Chagas Cardiomyopathy. International Journal Cardiovascular Sciences. Rio de Janeiro, 2020; 33(6):697-704.

14. NOGUEIRA SS, et al. Challenges of Immunosuppressive and Antitrypanosomal Drug Therapy after Heart Transplantation in Patients with Chronic Chagas Disease: A Systematic Review of Clinical Recommendations. Transplantation Reviews, 2018; 32(3): 157-167.

15. POLTRONIERI NV, et al. Não adesão medicamentosa nos pacientes transplantados cardíacos. Revista da Escola de Enfermagem da USP, 2020; 54: 1-9.

16. RADISIC MV, REPETTO SA. American trypanosomiasis (Chagas disease) in solid organ transplantation. Transplant Infectious Disease, 2020; 22(6): 1-12.

17. RODRIGUES BA, et al. Reativação da doença de Chagas pós-transplante cardíaco. Revista Eletrônica Acervo Científico, 2020.

18. ROSCOE A, et al. Chagas Cardiomyopathy: A Comprehensive Perioperative Review. Journal of Cardiothoracic and Vascular Anesthesia, 2018; 32(6): 2780-2788.

19. SANTOS E, FALCÃO LM. Chagas cardiomyopathy and heart failure: From epidemiology to treatment. Portuguese Society of Cardiology, 2019.

20. SIMÕES MV, et al. Cardiomiopatia da doença de Chagas. International Journal of Cardiovascular Sciences, 2018; 31(2): 173-189.

21. VIEIRA JL, et al. Chagas cardiomyopathy in Latin America review. Current cardiology reports, 2019; 21 (2): 8. 